\title{
Comparison of Ultem 9085 Used in Fused Deposition Modelling (FDM) with Polytherimide Blends
}

\author{
Gianluca Cicala ${ }^{1,2, *(\mathbb{D})}$, Giulia Ognibene ${ }^{1,2}$, Salvatore Portuesi ${ }^{1,2}$, Ignazio Blanco ${ }^{1,2}$ (D), \\ Mario Rapisarda ${ }^{1,2}$, Eugenio Pergolizzi ${ }^{1,2}$ and Giuseppe Recca ${ }^{3}$ \\ 1 Dipartimento di Ingegneria Civile e Architettura, Università di Catania, Viale Andrea Doria 6, \\ 95125 Catania, Italy; giuliaognibene@live.com (G.O.); salvo.p90@live.it (S.P.); iblanco@dii.unict.it (I.B.); \\ rapisardamario@yahoo.com (M.R.); euper@hotmail.com (E.P.) \\ 2 UdR-Catania Consorzio National Interuniversity Consortium of Materials Science and Technology (INSTM), \\ Viale Andrea Doria 6, 95125 Catania, Italy \\ 3 Institute for Polymers, Composites and Biomaterials (ICPB), National Research Council (CNR), \\ Via Paolo Gaifami 17, 95125 Catania, Italy; giuseppe.recca@cnr.it \\ * Correspondence: gcicala@unict.it
}

Received: 22 January 2018; Accepted: 10 February 2018; Published: 12 February 2018

\begin{abstract}
Polyetherimide (PEI) blends modified by either polycarbonate (PC) or polyethylene terephthalate glycol-modified (PETG) were prepared. The latter modifier (PETG) was an industrial grade widely used for fused deposition modelling (FDM) printing. PEI blends were compared to Ultem 9085, which is the standard PEI grade for FDM printing in advanced applications. All the blends were thoroughly characterized in terms of their rheological, morphological, thermomechanical and tensile properties. Ultem 9085 showed improved rheology for processing over standard PEI. $\mathrm{PEI} / \mathrm{PC}$ blends with $10 \mathrm{wt} \%$ of modifier developed here closely matched the viscosity behavior of Ultem 9085. On the other hand, the blends with low PC content (i.e., less than $20 \mathrm{wt} \%$ ) outperformed Ultem 9085 in terms of thermal and tensile properties. When PETG was added, similar tensile properties to Ultem 9085 were found. The immiscibility for PC contents higher than $20 \mathrm{wt} \%$ deteriorated the tensile properties, making it less attractive for applications, although melt viscosity decreased further for increasing PC contents.
\end{abstract}

Keywords: thermoplastic blends; additive manufacturing; fused deposition modelling; mechanical properties

\section{Introduction}

Additive manufacturing (AM) is gaining increasing importance in industry not only for prototyping, but, in many cases, for the production of functional parts [1]. The global market for AM products and services has grown into a $\$ 1.3$ billion industry, and it is estimated to grow to over $\$ 5$ billion by 2020 [1]. Among the different AM techniques, the filament based technology referred as the Fused Deposition Modelling (FDM) is the most widely used and it is also recognized to be the best AM technique for functional parts [2]. To further push the application of FDM for functional applications, the development of engineering polymers for FDM is of paramount importance.

Stratasys (Eden Praire, MN, USA) is the leading company that, founded by Scott Crump, developed the original FDM concepts, and it is now producing the reference industrial machine for FDM under the tradename FORTUS. These machines can operate with acrylonitrile butadiene styrene (ABS), nylon (Ny), polycarbonate (PC), PC-ABS, ASA, polyetherimide (PEI), and polyphenylsulfone (PPSF).

Stratsys offers three choices as high performance filaments: Ultem 1010, Ultem 9085 and Polyphenylsulfone (PPSF). Both the Ultem grades are based on polyetherimide (PEI) and they are 
certified for use in the automotive, medical and aerospace fields. Ultem 1010 is a pure PEI, while Ultem 9085 is reported to be a mixture of PEI and polycarbonate copolymer blend incorporated for improved flow [3].

Zaldivar et al. [3] showed recently that Ultem 9085 printed specimen mechanical properties are significantly affected by build orientation. The strength utilization in terms of FDM/injection molded performance can vary from $85.8 \%$ for edge printed specimens to $46.5 \%$ for up printed samples. This means that the final properties of the FDM printed part are highly anisotropic. The lower performances, compared to injection molded specimens, were the result of the voids in FDM printed parts between fused filaments. Motaparti et al. [4] recently showed that the strength of a thermoplastic interface within FDM part is directly proportional to the intermolecular diffusion across the interface between the fused filaments. The relevance of bond quality between adjacent filaments depends on printing parameters, but, also, on the melt viscosity of the polymer used for the filaments. However, to the best of our knowledge, this aspect has been properly investigated in the literature by a few studies only from McIlroy et al. [5,6].

The aim of the present paper is twofold: to discuss the rheological, morphological and thermomechanical properties of Ultem 9085; to develop laboratory made PEI/PC and PEI/PETG blends, which can compete with Ultem 9085. In order to achieve the latter objective, the properties of the injection molded blends are reported in comparison to pellets obtained from Ultem 9085.

\section{Experimental}

\subsection{Materials and Methods}

\subsubsection{Materials}

An industrial grade polycarbonate (PC), named Xantar, was obtained from Mitsubishi Engineering Plastics, Düsseldorf, Germany. An industrial grade polyethylene terephthalate glycol-modified (PETG) branded as XT was purchased as pellets from Colofabb, Belfeld, the Netherlands. XT is a PETG grade specifically developed to be used as filament for FDM printing. An industrial grade polyetherimide (PEI) was purchased from Luvocomm, Hamburg, Germany. For comparison purposes, the filament Ultem 9085 by Stratasys was tested. All of the materials were used as received.

\subsubsection{Blends Compounding and Specimens Manufacturing}

PEI based blends were melt mixed in a batch mixer (Brabender 50 EHT, Brabender \& Co., Duisburg, Germany) controlled by a Lab-Station. The torque and the temperature were measured during all the tests. The Brabender Software Winmix was used to record the data. The content of PC in the blends varied from $0 \mathrm{wt} \%$ to $40 \mathrm{wt} \%$. The blends with PETG were prepared at two PETG contents only, i.e., $5 \mathrm{wt} \%$ and $10 \mathrm{wt} \%$. The laboratory-made blends with PEI and PC were dry blended in the desired quantity, and, then, a total of $45 \mathrm{~g}$ of the dry blend were introduced in the preheated chamber of the batch mixer at $300{ }^{\circ} \mathrm{C}$. For PEI/PETG blends, the mixer temperature was lowered to $280{ }^{\circ} \mathrm{C}$. The rotor speed was kept at $50 \mathrm{rpm}$ for all the tests. The samples were extracted after $10 \mathrm{~min}$ from the loading in the mixer and left to cool at room temperature.

PEI and PEI/PC injection molded specimens were fabricated using a microinjection molder (Megatech H7/18-1, TecnicaDueBi, Fabriano (AN), Italy) with the settings reported in Table 1. The specimens were allowed to cool down in the mold for 5 min before extraction. Ultem 9085 was pelletized and then processed by injection molding to prepare specimens for comparison testing with PEI based blends. Ultem 9085 injection molded specimens were fabricated using a microinjection molder (Megatech H7/18-1) following the injection settings reported in Table 1. Incremental ratios of PC and PETG were added from 5 to $40 \mathrm{wt} \%$ and 5 to $10 \mathrm{wt} \%$ for PC and PETG, respectively. The choice to add less than $50 \mathrm{wt} \%$ of PC is due to the overall lower thermal performances of PC, which can impair the final thermal properties of the blends. Similarly, a lower amount of PETG was 
added as this polymer has even lower thermal properties than PC. This point is relevant as PEI based filaments are the preferred choice when high glass transition temperature or high head distortion temperature are requirements.

Table 1. Polymer blend ratios prepared by injection molding alongside with the parameters for processing.

\begin{tabular}{|c|c|c|c|c|c|c|c|}
\hline \multirow{2}{*}{ CODE } & PEI & PC & T1 & $\mathrm{T} 2$ & T3 & $\mathrm{T} 4$ & Pressure \\
\hline & $(w t \%)$ & $(w t \%)$ & $\left({ }^{\circ} \mathrm{C}\right)$ & $\left({ }^{\circ} \mathrm{C}\right)$ & $\left({ }^{\circ} \mathrm{C}\right)$ & $\left({ }^{\circ} \mathrm{C}\right)$ & (bar) \\
\hline PC100 & 0 & 100 & 220 & 280 & 300 & 40 & 30 \\
\hline PC0 & 100 & 0 & 320 & 350 & 350 & 60 & 90 \\
\hline PC5 & 95 & 5 & 320 & 350 & 350 & 60 & 90 \\
\hline PC10 & 90 & 10 & 320 & 350 & 350 & 60 & 90 \\
\hline PC20 & 80 & 20 & 320 & 350 & 350 & 60 & 90 \\
\hline PC 30 & 70 & 30 & 320 & 350 & 350 & 60 & 90 \\
\hline \multirow[t]{4}{*}{ PC40 } & 60 & 40 & 320 & 350 & 350 & 60 & 90 \\
\hline & \multicolumn{2}{|c|}{ PEI Stratasys } & 300 & 330 & 330 & 60 & 90 \\
\hline & PEI & PETG & T1 & $\mathrm{T} 2$ & T3 & T4 & Pressure \\
\hline & $(w t \%)$ & $(w t \%)$ & $\left({ }^{\circ} \mathrm{C}\right)$ & $\left({ }^{\circ} \mathrm{C}\right)$ & $\left({ }^{\circ} \mathrm{C}\right)$ & $\left({ }^{\circ} \mathrm{C}\right)$ & (bar) \\
\hline PETG5 & 95 & 5 & 320 & 330 & 330 & 60 & 90 \\
\hline PETG10 & 90 & 10 & 320 & 330 & 330 & 60 & 90 \\
\hline
\end{tabular}

\subsection{Characterization}

\subsubsection{Mechanical Testing}

Tensile and flexural specimens were prepared accordingly to ASTM D638 [7] and ASTM D790 [8], respectively. The tensile and flexural properties of the printed materials were investigated testing five samples for each kind of the material under study. Tensile properties of printed specimens were measured by using an Instron 5985 universal testing machine (Instron, Milano, Italy), equipped with a load cell of $10 \mathrm{kN}$. Specimens were tested at a constant speed of $5 \mathrm{~mm} / \mathrm{min}$ while compliance correction was used. System control and data analysis were performed using Instron's Blue Hill v.3.

Differences in mechanical results were statistically analyzed by one-way analysis of variance (ANOVA) using Minitab 17 software. To identify which groups were significantly different from other groups, means comparison was done using the Fisher's test with a 95\% confidence level.

\subsubsection{Scanning Electron Microscopy (SEM)}

Criofractured surfaces were analyzed with an EVO Scanning Electron Microscope (EVO-SEM, Zeiss, Cambridge, UK). An acid mixture (sulfuric acid/distilled water (3:2)) was used to enhance the phase contrast of the PEI/PC blend. The effect of the etching procedure was to increase the contrast between the thermoplastic phases. The etching treatment was carried out by the immersion of the samples in the acid mixture, followed by a stirring time varying from 5 to $20 \mathrm{~min}$. The samples were always washed with water after etching, and were then sputtered with gold. All the samples were gold sputtered up to a thickness of $20 \mathrm{~nm}$ by means of a Emitech K-550 sputter coater (Ashford, Kent, $\mathrm{UK})$. An accelerating voltage of $15 \mathrm{kV}$ was used to collect the micrographs.

\subsubsection{Dynamic Mechanical Analysis (DMA)}

The viscoelastic behavior of the solid specimens was investigated using a DMA instrument (TRITEC by Triton Technology, City, United Kingdom) by single cantilever geometry and using samples of size $(10 \times 5 \times 2) \mathrm{mm}$. The tests were carried out at 1 and $10 \mathrm{~Hz}$ with a $2{ }^{\circ} \mathrm{C} / \mathrm{min}$ heating rate ranging from $25^{\circ} \mathrm{C}$ to $250{ }^{\circ} \mathrm{C}$. 


\subsubsection{Rheological Analysis on Polymer Blends}

Rheological analyses were performed with a rotational rheometer ARES by TA Instruments (Milan, Italy) equipped with parallel plates of $25 \mathrm{~mm}$ diameter. The polymer samples were charged on the pre-heated lower plate of the rheometer, and the upper plate was then lowered to obtain a gap of $1.2 \mathrm{~mm}$. The melting of sample occurred and the excess of the molten polymer was removed with a clean wood spatula. The frequency sweep test was carried out at $350{ }^{\circ} \mathrm{C}$ and the frequency varied from 0.1 to $100 \mathrm{rad} / \mathrm{s}$. The strain was fixed at $5 \%$ because some preliminary runs, at various frequencies, showed linear viscoelastic behavior of the blends in these experimental conditions.

\section{Results and Discussion}

\subsection{Rheological Properties of Ultem 9085 versus PEI Based Blends}

The rheological properties of the pure blend's components were analyzed first. The linear viscoelastic properties of pure polymers have been investigated using frequency sweep measurements with low amplitude strain (i.e., $5 \%)$. The complex viscosity $\left(\eta^{*}\right)$, the storage modulus $\left(G^{\prime}\right)$ and the loss modulus $\left(\mathrm{G}^{\prime \prime}\right)$ as a function of frequency for pure PEI and Ultem 9085 are showed in Figure 1. For both polymers, the complex viscosity decreases with increasing frequencies. However, Ultem 9085 (Figure 1a) presented a lower viscosity compared to unmodified PEI over the entire frequency range. In addition to that, Ultem 9085 displayed a markedly shear thinning behavior compared to the unmodified PEI. The complex viscosity versus frequency of the unmodified PEI used in this study resembled the behavior reported by Nobile et al. [9] for standard Ultem 1000.
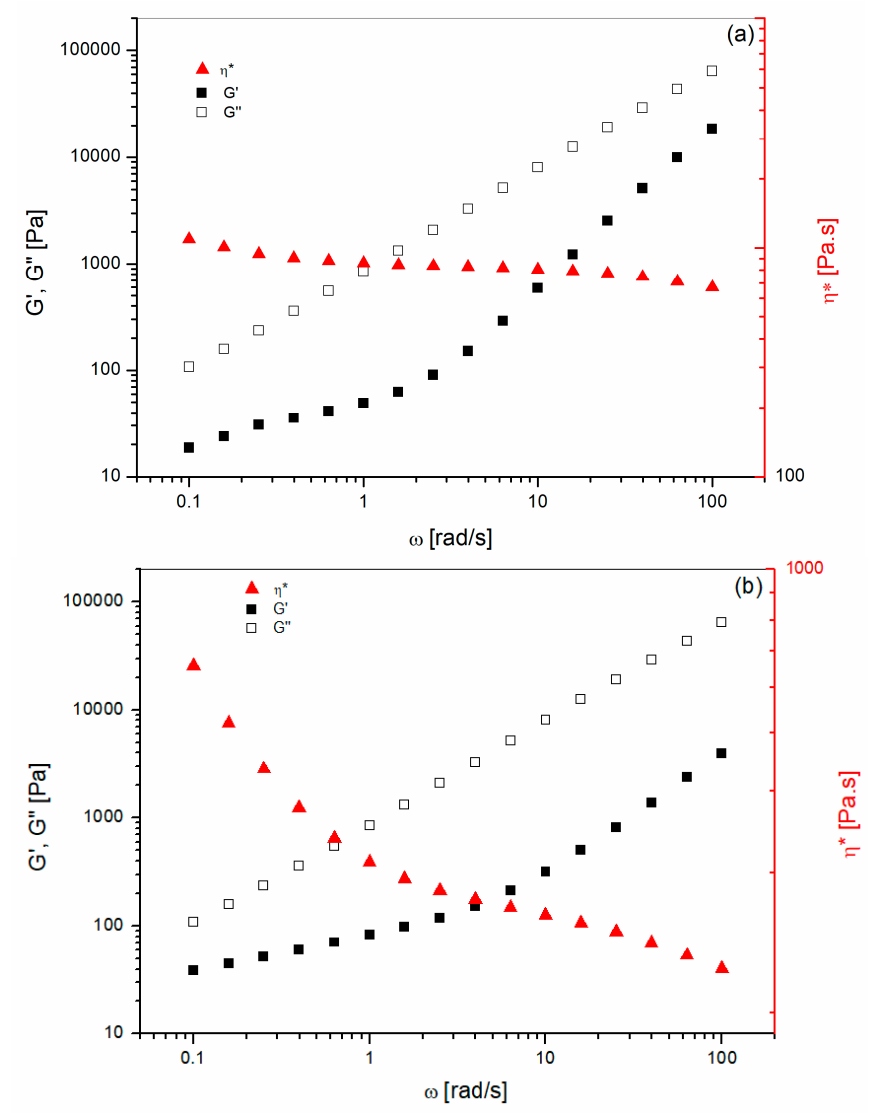

Figure 1. Isothermal rheology at $350{ }^{\circ} \mathrm{C}$ for: (a) PEI; (b) Ultem 9085.

The complex viscosities for the modified PEI blends with different PC contents are displayed in Figure 2. 


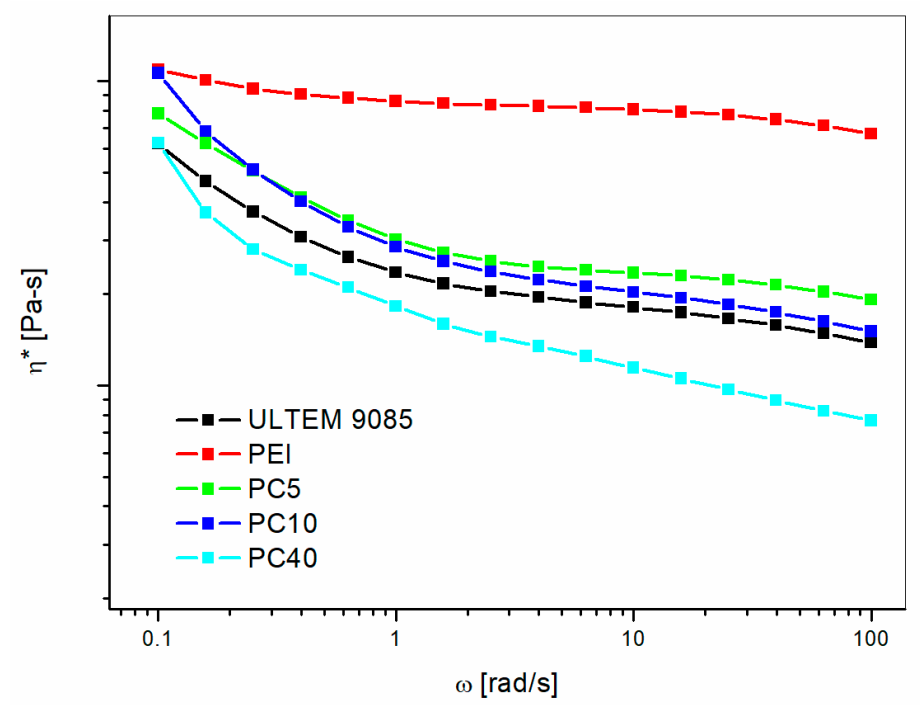

Figure 2. Isothermal rheology at $350{ }^{\circ} \mathrm{C}$ for PEI/PC blends with different content of PC.

The graph shows that adding PC or PETG resulted in decreasing viscosities with increasing modifier contents in the high frequencies range. Nobile et al. [9] showed, adding thermotropic liquid crystalline polymer, that the viscosity of PEI is lowered by the inclusion of a low viscosity second phase. Similar results were obtained by Ramiro et al. [10] by measuring the exit melt pressure directly from the extruder for PEI modified by Poly(trimethyleneterephatalate) (PTT). All of these findings support the conclusion that adding PC or PETG leads to improvements in melt processing of PEI based blends. These results were further confirmed by the torque measured during melt blending in the static mixer (Figure 3).

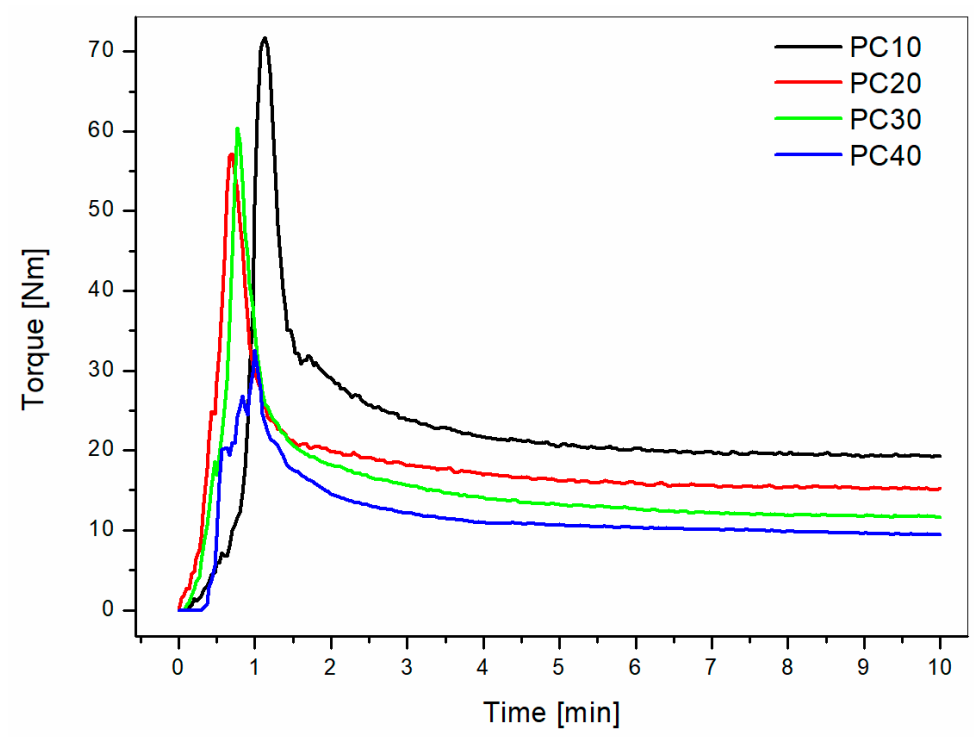

Figure 3. Torque versus time curves for PEI/PC blends mixed at $300{ }^{\circ} \mathrm{C}$ for different $\mathrm{PC}$ contents.

The clear effect of PETG to lower the processing window of PEI was demonstrated by the preparation of mixed blends at $280^{\circ} \mathrm{C}$ in the static mixer, while injection molded samples were manufactured at $320^{\circ} \mathrm{C}$ instead of $350{ }^{\circ} \mathrm{C}$ that was used for PEI/PC. 


\subsection{Dynamic Mechanical Analysis of Ultem 9085 versus PEI Based Blends}

Storage modulus $\left(E^{\prime}\right)$, loss modulus $\left(E^{\prime \prime}\right)$ and loss factor (Tan $\delta$ ) versus temperature plots for the unmodified PEI and for Ultem 9085 are reported in Figure 4. The unmodified PEI showed a single clear Tan $\delta$ peak centered at $217^{\circ} \mathrm{C}$, while, for Ultem 9085 , a wide peak at $185^{\circ} \mathrm{C}$ and a shoulder at $140{ }^{\circ} \mathrm{C}$ were observed. The presence of this shoulder for Ultem 9085 could be the result of the presence of a second phase dispersed in the polymer as stated by Zaldivar et al. [3]. Similar behavior was observed in the loss modulus curves. The unmodified PEI showed the standard behavior reported for PEI industrial grades like Ultem 1000, while similar shifts to lower temperatures for the main Tan $\delta$ peak were reported previously for blends PEI mixed with compatibilized second phase [10].
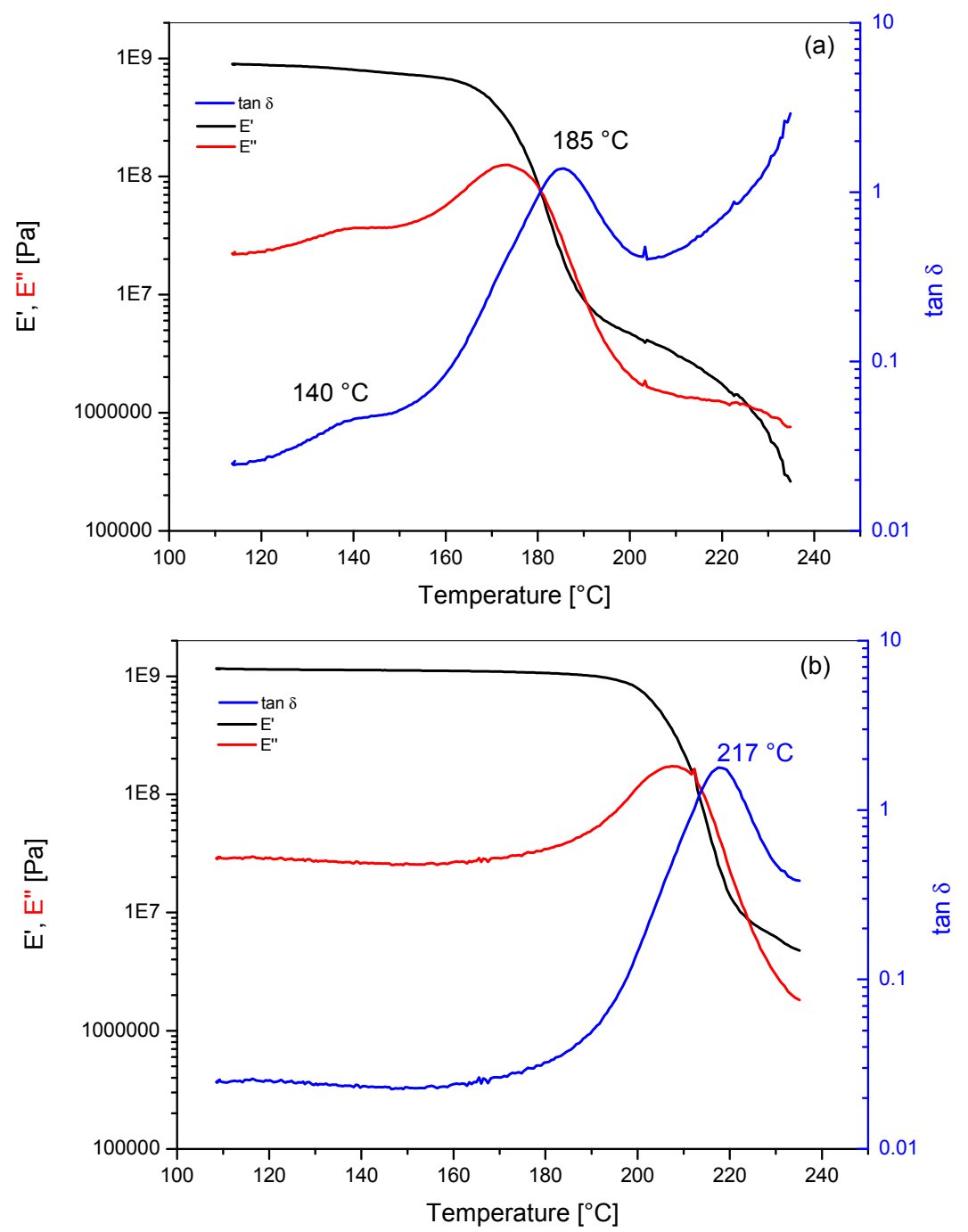

Figure 4. Dynamic mechanical analysis for PEI based systems: (a) Ultem 9085 and (b) PEI.

The effects on Tan $\delta$ and storage modulus ( $\left.\mathrm{E}^{\prime}\right)$ of blending PC or PETG with PEI are showed in Figures 5 and 6, respectively. The Tan $\delta$ curves showed two distinct peaks for PEI/PC blends with PC content higher than $10 \mathrm{wt} \%$ : one main peak centered between $210{ }^{\circ} \mathrm{C}$ and $217^{\circ} \mathrm{C}$; a smaller Tan $\delta$ peak centered between $143^{\circ} \mathrm{C}$ and $148^{\circ} \mathrm{C}$. Pure polycarbonate (PC) showed one single Tan $\delta$ peak at $150{ }^{\circ} \mathrm{C}$ in correspondence to its glass transition temperature (Figure 7). The blends modified with PETG showed one relaxation peak only but for decreasing temperatures with increasing PETG content. 


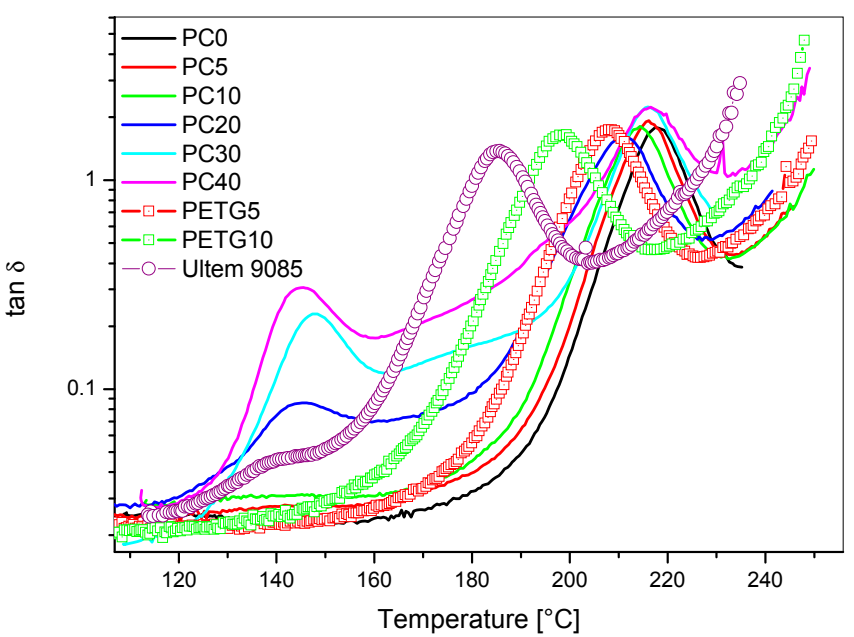

Figure 5. Tan $\delta$ versus temperature for PEI/PC and PEI/PETG blends compared to Ultem 9085: effect of PC and PETG content.

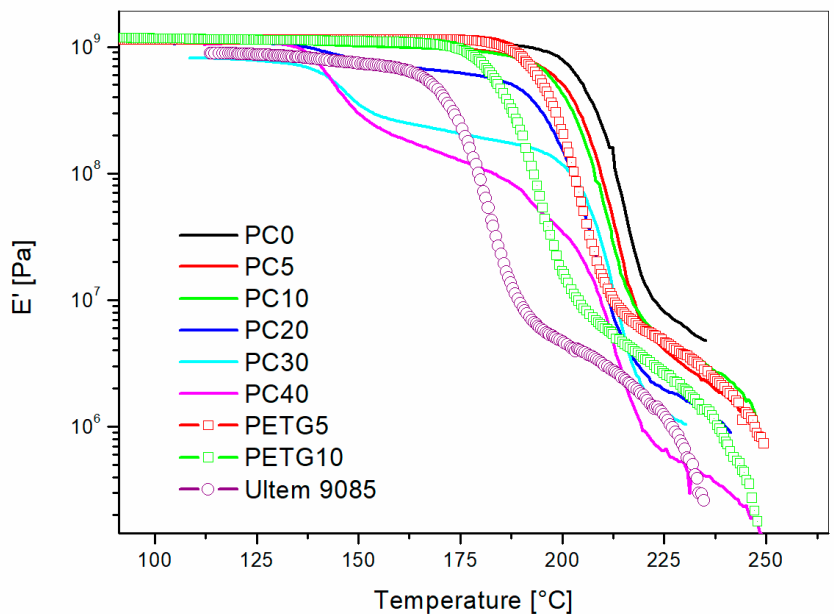

Figure 6. Storage Moudulu ( $\left.\mathrm{E}^{\prime}\right)$ versus temperature for PEI/PC and PEI/PETG blends compared to Ultem 9085: effect of PC and PETG content.

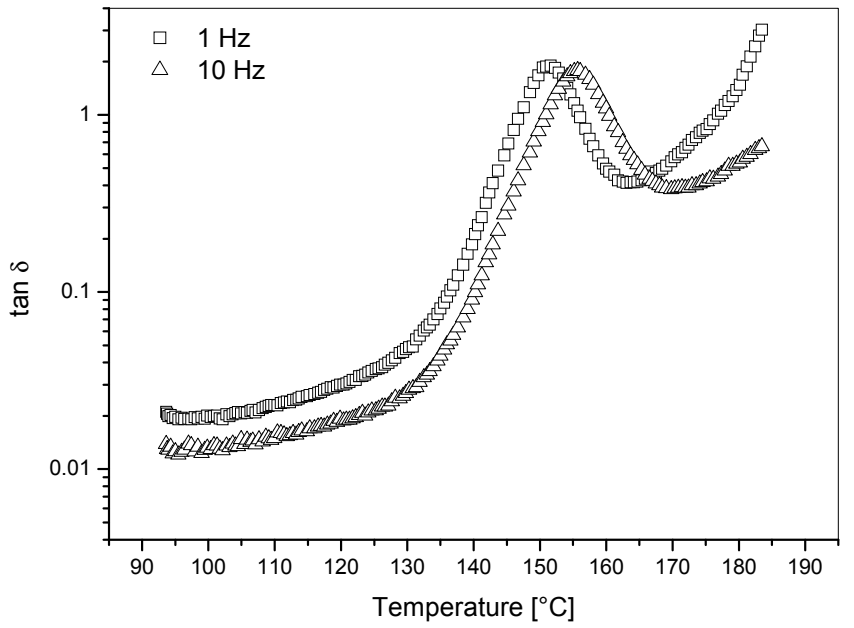

Figure 7. Tan $\delta$ versus temperature for PC used as modifier in PEI/PC blends tested at two different frequencies (i.e., $1 \mathrm{~Hz}$ and $10 \mathrm{~Hz}$ ). 
The presence of two Tan $\delta$ peaks in the blends with PC content higher than $10 \mathrm{wt} \%$ was the result of the immiscibility between PEI and PC. The shift of Tg's for PEI-rich phases in the PEI/PC blends is reported as a function of PC weight content in Figure 8.

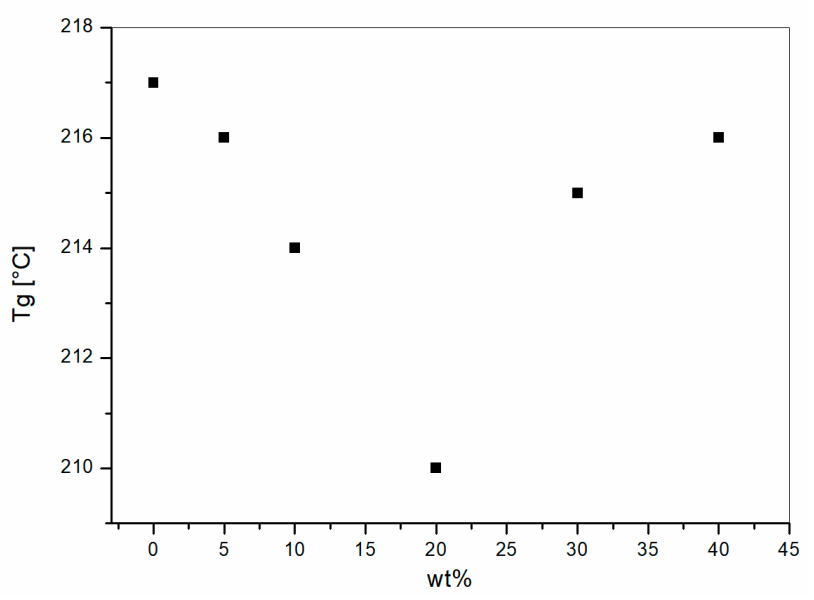

Figure 8. Glass Transition Temperature (Tg) versus PC content for the PEI rich phase (data extrapolated from peak analysis of Figure 6).

The glass transition trend for different PC content, which were observed previously [11,12], can be explained by the presence of residual PC dispersed in the PEI-rich phase at high PEI compositions. However, the shift measured are smaller than those observed for PEI/PETG. In addition to that, PEI/PETG blends showed one single Tan $\delta$ peak for both the PETG concentrations analyzed, thus leading to the conclusion that PETG had an enhanced miscibility with PEI compared to PC. Several authors demonstrated that PEI is miscible with PET and PBT polymers [12,13]; therefore, PETG could be a miscible polymer with PEI.

The comparison of the storage modulus $\left(\mathrm{E}^{\prime \prime}\right)$ versus temperature for all the PEI/PC blends showed clear modulus drops for temperatures lower than $175^{\circ} \mathrm{C}$ for the blends with PC content higher than $20 \mathrm{wt} \%$. The blends with $5 \mathrm{wt} \%$ and $10 \mathrm{wt} \%$ of PC showed modulus drops only for temperatures higher than $190^{\circ} \mathrm{C}$. The $\mathrm{E}^{\prime}$ values measured at $160^{\circ} \mathrm{C}$ and $185^{\circ} \mathrm{C}$ are reported in Figure 9 for all the $\mathrm{PEI} / \mathrm{PC}$ blends and for the Ultem 9085.

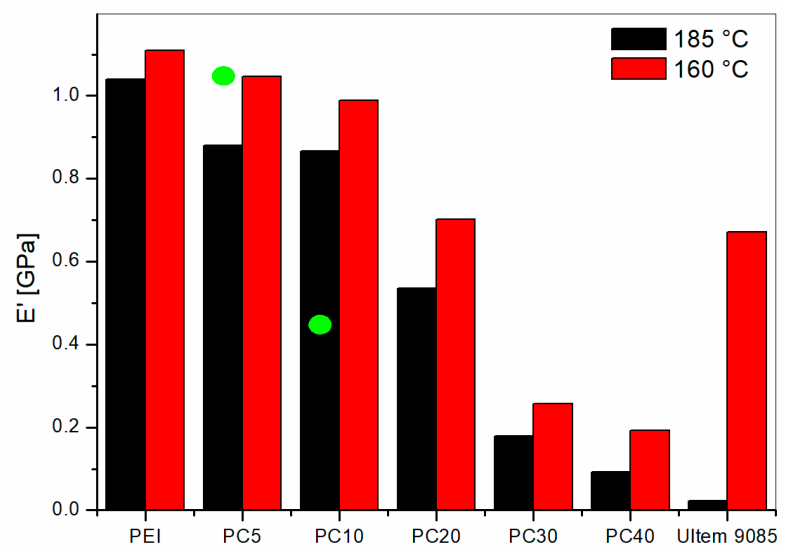

Figure 9. Storage Modulus values calculated at $165^{\circ} \mathrm{C}$ and $185^{\circ} \mathrm{C}$ from graph of $\mathrm{E}^{\prime}$ versus temperature. Green circles represent the value for PEI/PETG blends.

$\mathrm{PEI} / \mathrm{PC}$ blends displayed higher storage modulus than for Ultem 9085 at $185{ }^{\circ} \mathrm{C}$. However, for the measurements at $160{ }^{\circ} \mathrm{C}$, the blends with PC contents up to $10 \mathrm{wt} \%$ showed similar or 
higher storage modulus compared to Ultem 9085. These systems, which showed one Tan $\delta$ peak only, were characterized by a phase separation that did not allow for the formation of PC-rich phases, greatly impacting the storage modulus of the blends. The PEI/PC with enhanced lower Tan $\delta$ peaks showed higher modulus drops at $160^{\circ} \mathrm{C}$, which lead to a smaller modulus compared to Ultem 9085. The blend PC20 showed a transitional behavior with modulus at $160{ }^{\circ} \mathrm{C}$ similar to Ultem 9085 . Those results were further supported by the microscopic investigation of the fractured surface reported below.

PEI/PETG blends showed a single modulus drop. The storage modulus values at $185{ }^{\circ} \mathrm{C}$ for PETG5 and PETG10 were 1.03 and $0.46 \mathrm{GPa}$, respectively. These values are displayed as green circles in Figure 9 for comparison with PEI/PC blends. The graph shows that, for low PETG content (i.e., 5 wt \%), the modulus of the blend is higher compared to the PC5, but, as the PETG content is increased up to $10 \mathrm{wt} \%$, the modulus was halved compared to that of PC10. This difference was due to the increased content of PETG dispersed homogenously in the PEI matrix for PETG10 blend. However, if the comparison is made between PEI/PETG and Ultem 9085, the storage modulus at $185^{\circ} \mathrm{C}$ is always higher for the PETG modified blends.

\subsection{Morphological Analysis of Ultem 9085 versus PEI/PC Blends}

The morphology of PEI/PC blends was studied on criofractured surfaces using a scanning electron miscroscopy (SEM). The micrographs of Ultem 9085 are shown in Figure 10 for both etched and non-etched specimens. No peculiar morphology is displayed on the non-etched surface (Figure 10a). For this reason, we performed an acid etching on the surface of the sample and the result is shown in Figure $10 \mathrm{~b}$. The analysis on the etched surface revealed a particulate morphology with a well dispersed phase separation of small (about $250 \mathrm{~nm}$ ) particles. This result is coherent with the observed Tan $\delta$ curve, which showed a main peak and a shoulder at low temperature.

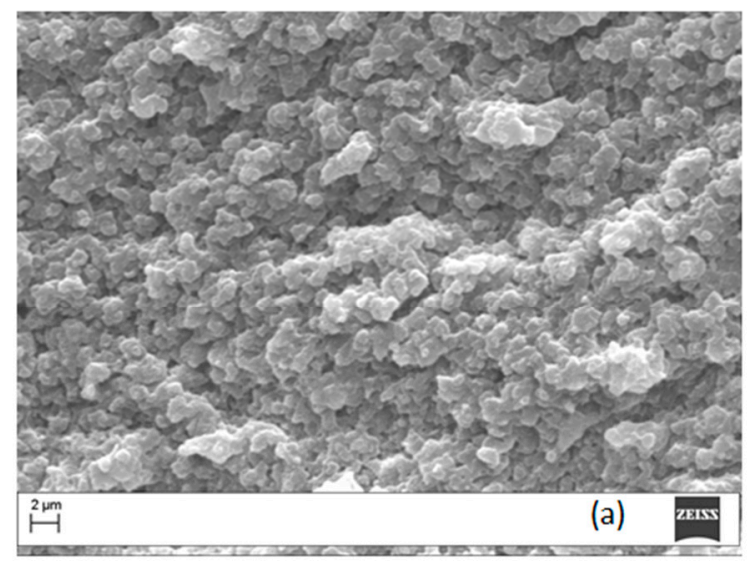

Non etched

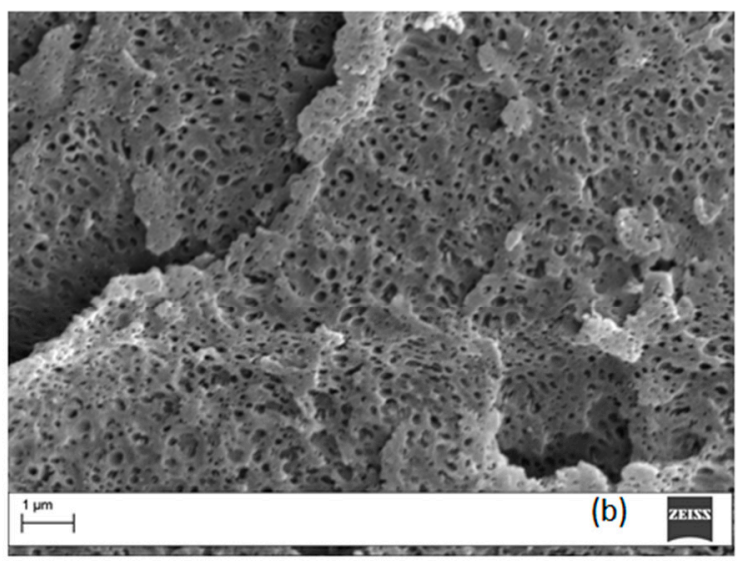

Etched

Figure 10. Micrographs of crio-fractured Ultem 9085 samples before (a) and after (b) chemical etching (magnification $20,000 \times$ ).

Similar analysis was carried out on the PEI/PC blend with 20 wt \% of PC and the results, for different etching times, are shown in Figure 11. The blend showed clear phase separation for all of the etched surfaces. However, after 20 min of etching, the presence of particulate was clearer. In order to obtain enhanced phase contrast, all of the PEI modified blends were analyzed applying the etching procedure. 


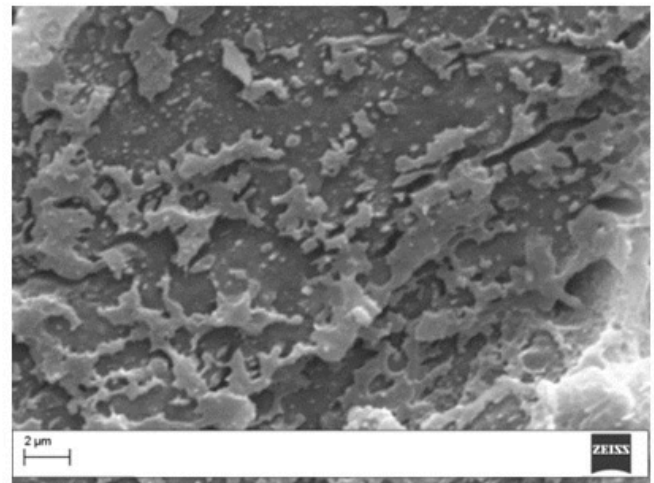

(a)

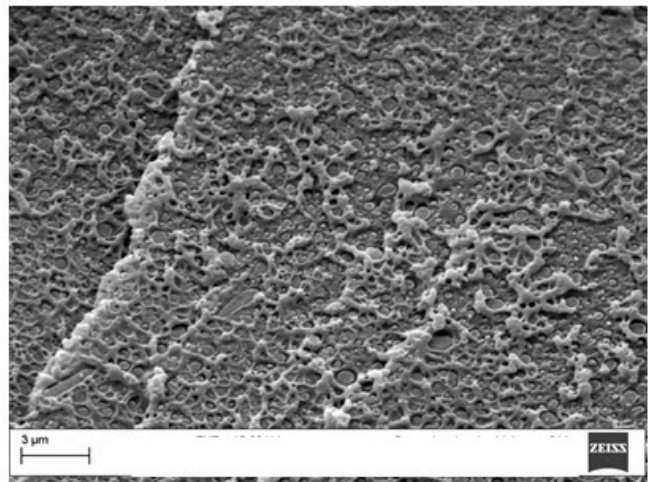

(c)

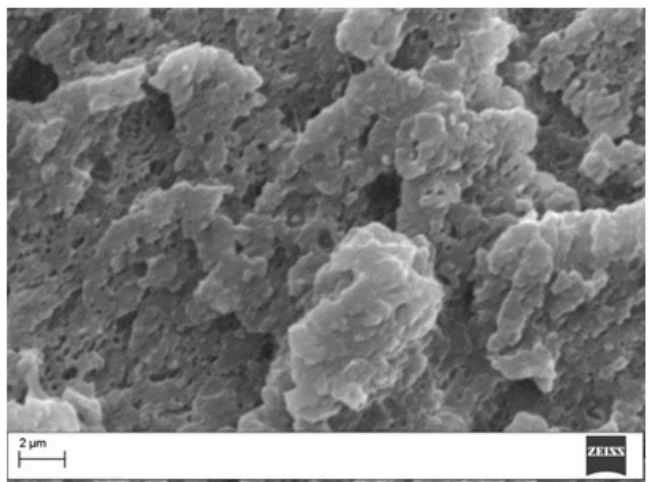

(b)

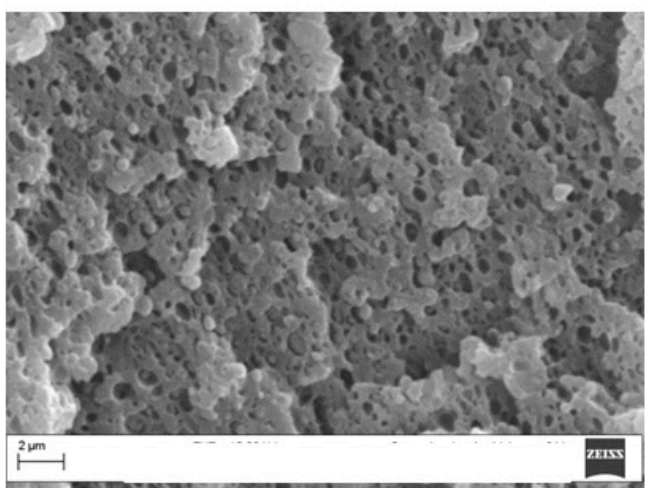

(d)

Figure 11. Micrographs of crio-fractured PEI/PC blends containing $20 \mathrm{wt} \%$ of PC for different etching time (magnification 10,000×): (a) $5 \mathrm{~min}$; (b) $10 \mathrm{~min}$; (c) $15 \mathrm{~min}$; (d) $20 \mathrm{~min}$.

Figure 12 reports the morphology of the blends with different contents of PC while Figure 13 shows the SEM analysis on PETG modified blends.
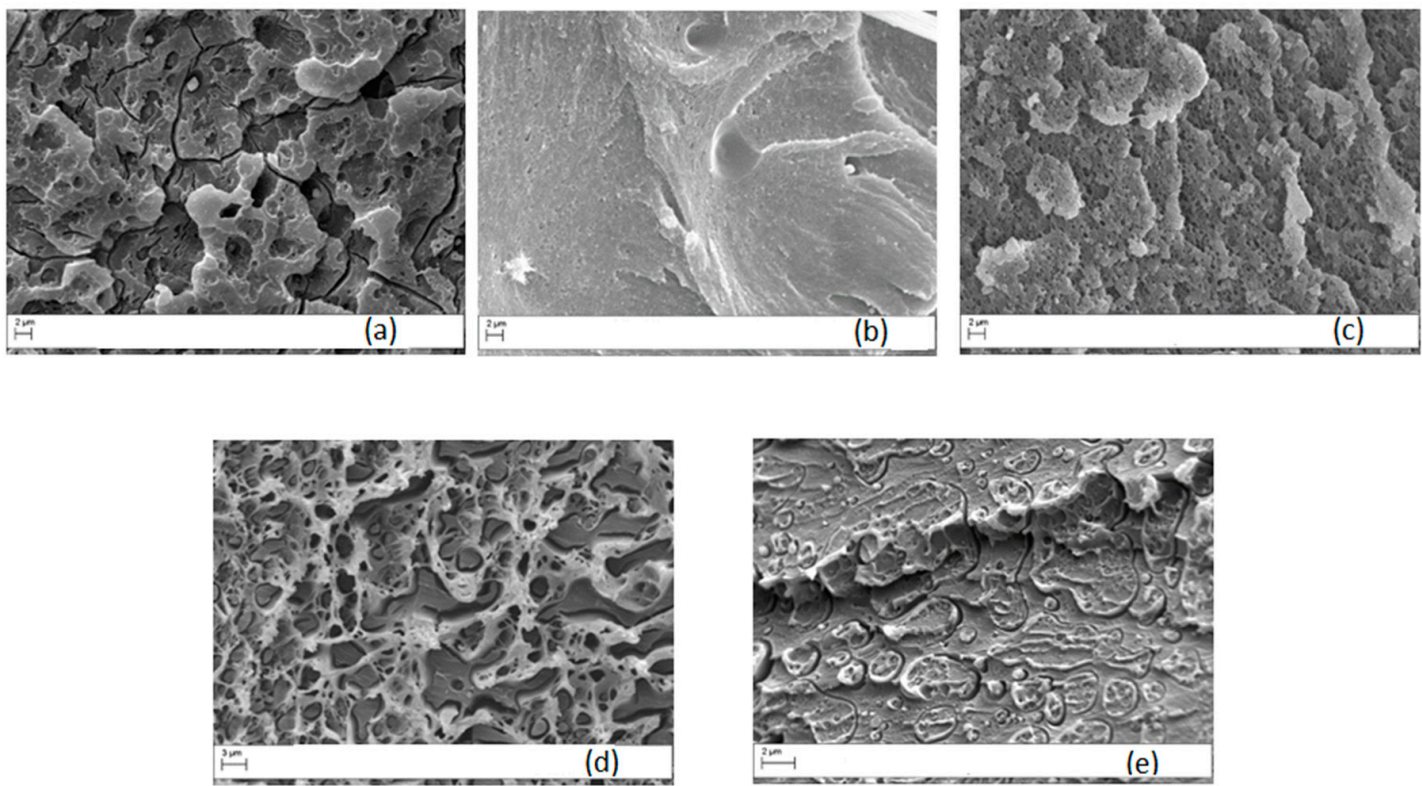

Figure 12. Micrographs of crio-fractured PEI/PC blends for different PC content (magnification 10,000×): (a) $5 \mathrm{wt} \%$; (b) $10 \mathrm{wt} \%$; (c) $20 \mathrm{wt} \%$; (d) $30 \mathrm{wt} \%$; (e) $40 \mathrm{wt} \%$. 
The results of the SEM analysis confirmed the findings of DMA described above because, for PC content below 20 wt \%, no clear phase separation was observed at the resolution analyzed. When the PC content increased up to $20 \mathrm{wt} \%$, particulate morphology with PC-rich particles of about $0.3 \mu \mathrm{m}$ appeared. The blend with $30 \mathrm{wt} \%$ of PC showed a co-continuous morphology, while phase inversion was observed when PC content was $40 \mathrm{wt} \%$. In contrast to this behavior, when PETG was used as a modifier, morphologies similar to Ultem 9085 were observed as the result of the improved miscibility of PETG and PEI (Figure 13).
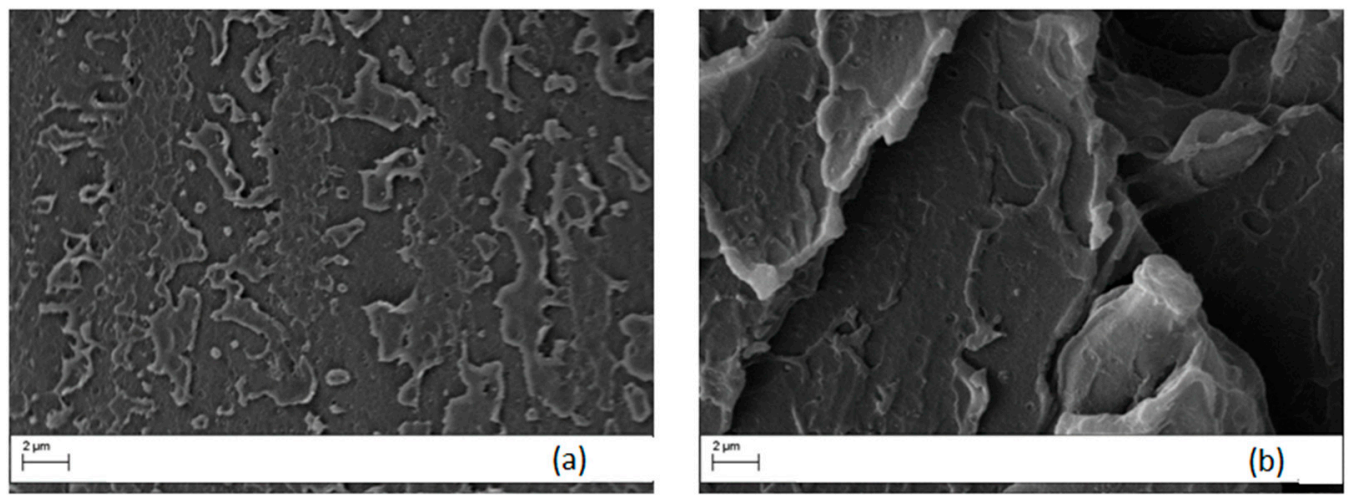

Figure 13. Micrographs of crio-fractured PEI/PETG blends for different PETG content (magnification 10,000×): (a) $5 \mathrm{wt} \%$; (b) $10 \mathrm{wt} \%$.

\subsection{Mechanical Properties of Ultem 9085 versus PEI/PC Blends}

The tensile properties for all the PEI blends and for Ultem 9085 were determined at room temperature. The results are summarized in Figure 14 for the tensile modulus and tensile yield strength. Ultem 9085 displayed a tensile modulus and a yield strength of $2.36 \pm 0.03 \mathrm{GPa}$ and $78.77 \pm 0.94 \mathrm{MPa}$, respectively. These values were higher than those reported in the technical literature for Ultem 9085 by Stratasys, but this is in accordance to what presented previously when comparing injection molded specimens to FDM samples [14]. The unmodified PEI used here showed tensile modulus and yield strength of $3.10 \pm 0.03 \mathrm{GPa}$ and $101.49 \pm 0.94 \mathrm{MPa}$, respectively. The values found are higher for unmodified PEI because it is a pure PEI rather than a blend as for Ultem 9085.

The PEI/PC blends showed a decreasing trend for both the modulus and strength with increasing values of PC content. However, for PC content up to $40 \mathrm{wt} \%$, the PEI/PC blends showed equal or higher tensile properties compared to Ultem 9085 samples. The experimental data for both tensile modulus and strength showed negative deviations from the rule of mixture. Ramiro et al. [15] showed similar behavior for PEI/PC blends, which were interpreted as the result of the slightly different orientation level of the components in the blends and in the pure state. Similar behavior is observed in the blends prepared here, which showed analogous morphologies to those reported by Ramiro et al. [15].

ANOVA analysis helped to shed more light on the influence of PC content. The tensile modulus means were all significantly different $(p<0.05)$ with the exception of the pairwise 20-30 $(p=0.333)$. The yield strength means seemed, accordingly to Fisher, not significantly different for the pairwise 0-5, 5-10 and 20-40. However, if the measured values were directly compared, only the 20-40 showed $p$-value higher than $0.05(0.728)$, thus confirming that the means are not statically different. The ANOVA analysis on the PEI/PETG blends revealed that the tensile modulus and yield strength of the systems with $5 \mathrm{wt} \%$ and $10 \mathrm{wt} \%$ were not statistically different with $p$-value of 0.307 and 0.809 , respectively. 

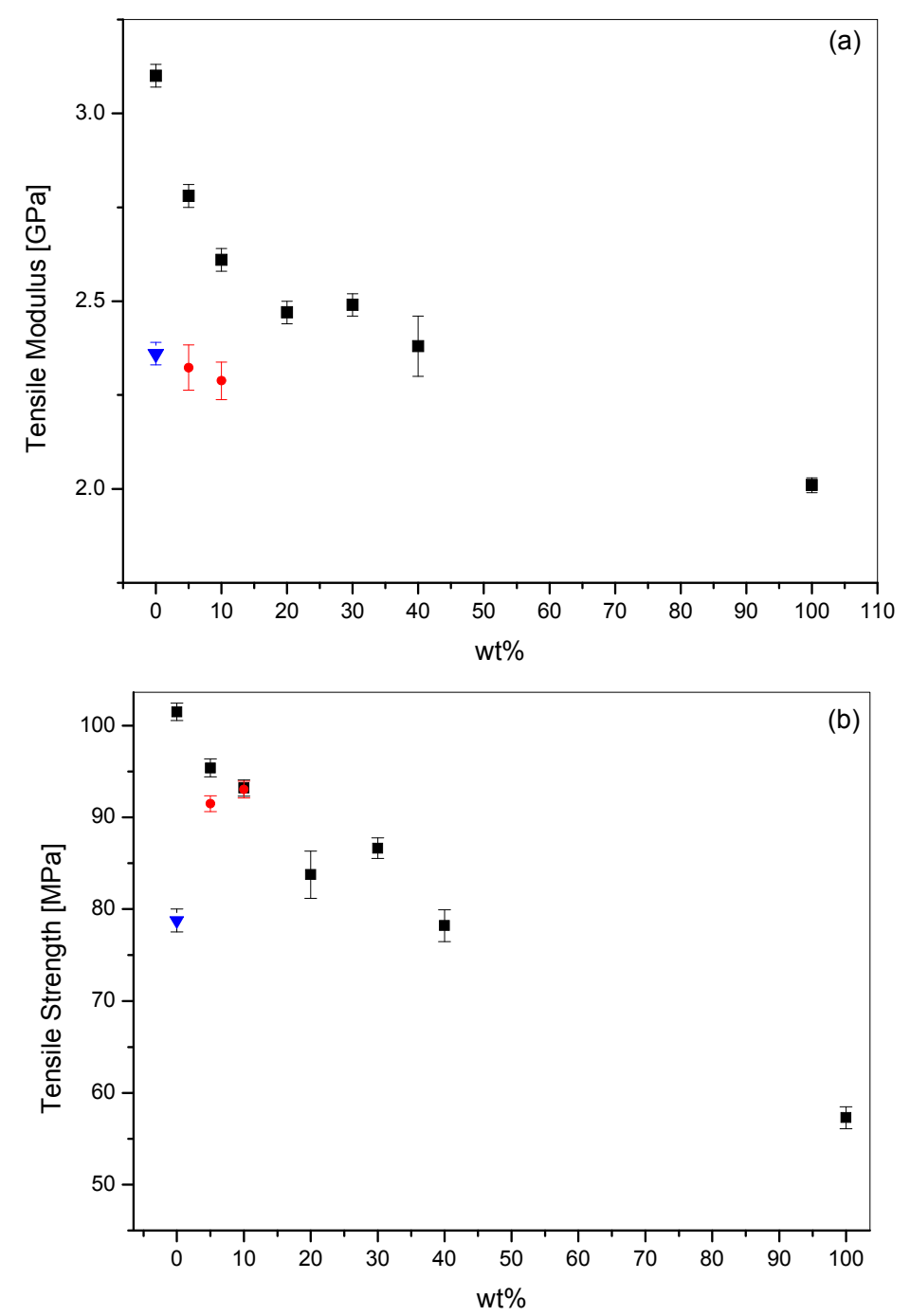

Figure 14. Effect of modifier content for PEI/PC (black) and PEI/PETG (red) blends compared to Ultem 9085 (blue) on tensile modulus (a) and tensile strength (b).

\section{Conclusions}

PEI based blends were prepared by melt mixing adding two different modifiers: PC and PETG. The PEI blends properties were measured and compared to Ultem 9085, which is a qualified PEI grade used by the leading company Stratasys for FDM.

Ultem 9085 showed a different viscosity behavior when compared to standard unmodified PEI. The viscosity for Ultem 9085 was lower thus with improved melt processing. Adding PC and PETG had similar effects on the viscosity leading to decreasing viscosity for increasing modifier contents. PC, in particular, when added at $10 \mathrm{wt} \%$ showed a complex behavior similar to Ultem 9085 . Therefore, the findings presented here demonstrated that PEI based blends with processing properties comparable to Ultem 9085 can be easily obtained by mixing standard PEI with a selected amount of PC.

DMA analysis showed a distinct different behavior for PEI modified by PC and PETG. The latter reduced the blend's glass transition temperature of $10{ }^{\circ} \mathrm{C}$ and $20{ }^{\circ} \mathrm{C}$ when $5 \mathrm{wt} \%$ and $10 \mathrm{wt} \%$ of modifier were added, respectively. SEM highlighted a homogenous morphology, which lead to the conclusion that miscible blends were formed upon mixing. Ultem 9085 showed high $\mathrm{Tg}$ reduction for the main phase, but, in this case, a small shoulder appeared at $140{ }^{\circ} \mathrm{C}$ and small particles were observed by SEM, which leads to the conclusion that this system is a compatibilized blend. On the contrary, PEI/PC blends showed glass transition temperature reductions of maximum $10^{\circ} \mathrm{C}$ for the 
blend with $20 \mathrm{wt} \%$ of PC. In addition to that, two single peaks were clearly showed for the blends with PC content higher than $10 \mathrm{wt} \%$ and SEM analysis confirmed the presence of biphasic morphologies for these blends. The phase separation was not clearly observed for PEI/PC blends with 5 wt \% and 10 wt \% of PC.

The tensile properties and the storage modulus at $160{ }^{\circ} \mathrm{C}$ and $185^{\circ} \mathrm{C}$ provided evidence that PEI blends with PC content lower than $20 \mathrm{wt} \%$ of PC behaved better than Ultem 9085 for all of the parameters considered. In terms of tensile yield strength, PEI/PC and PEI/PETG behaved similarly, but PEI/PC outperformed PETG based blends in terms of the tensile modulus. For PC content higher than $20 \mathrm{wt} \%$, the PEI/PC lost its advantages over Ultem 9085 showing higher storage modulus at $185{ }^{\circ} \mathrm{C}$ only.

Ultem 9085 filament is sold by Stratasys with $1.5 \mathrm{~kg}$ cartridge costing about $750 €$. From other filament producers [16] Ultem 9085 is sold at about $200 € / \mathrm{kg}$. However, unmodified ultem, such as the grade used in this paper, can be purchased in pellets at $50 € / \mathrm{kg}$ only, while PC is purchased at $20 € / \mathrm{kg}$. The data reported in this paper provided evidence that standard PEI can be modified with $10 \mathrm{wt} \%$ of PC to obtain a blend with promising properties to compete with Ultem 9085 in terms of similar melt processing properties but overall better performances. In addition to that, adding PC to PEI can further reduce the cost per kilogram, and, in turn, reduce by at least half the final price of the filaments.

Acknowledgments: Financial support: Bando Congiunto INSTM-Regione Lombardia 2016—Project IN-RL11 COMMAND-Materials for Additive Manufacturing and Italian Minister for University and Research (MIUR) for support to purchase equipment trough the grant PON BRIT.

Author Contributions: Gianluca Cicala conceived and designed the experiments; all authors performed the experiments; Gianluca Cicala and Ignazio Blanco analyzed the data; Gianluca Cicala wrote the paper.

Conflicts of Interest: The authors declare no conflict of interest.

\section{References}

1. Gardan, J. Additive manufacturing technologies: State of the art and trends. Int. J. Prod. Res. 2016, 54, 3118-3132. [CrossRef]

2. Turner, B.N.; Strong, R.; Gold, S.A. A review of melt extrusion additive manufacturing processes: I. Process design and modeling. Rapid Prototyp. J. 2014, 20, 192-204. [CrossRef]

3. Zaldivar, R.J.; Witkin, D.B.; McLouth, T.; Patel, D.N.; Schmitt, K.; Nokes, J.P. Influence of processing and orientation print effects on the mechanical and thermal behavior of 3D-Printed Ultem ${ }^{\circledR} ; 9085$ Material. Addit. Manuf. 2017, 13, 71-80. [CrossRef]

4. Motaparti, K.P.; Taylor, G.; Leu, M.C.; Chandrashekhara, K.; Castle, J.; Matlack, M. Experimental investigation of effects of build parameters on flexural properties in fused deposition modelling parts. Virtual Phys. Prototyp. 2017, 12, 1-14. [CrossRef]

5. Mcllroy, C.; Olmsted, P.D. Disentanglement effects on welding behaviour of polymer melts during the fused-filament-fabrication method for additive manufacturing. Polymer 2017, 123, 376-391. [CrossRef]

6. Mcllroy, C.; Olmsted, P.D. Deformation of an Amorphous Polymer during the Fused-Filament-Fabrication Method for Additive Manufacturing. J. Rheol. 2017, 61, 379-397. [CrossRef]

7. Standard Test Method for Tensile Properties of Plastics; ASTM D638; Publisher: ASTM International, West Conshohocken, PA, USA, 2014.

8. Standard Test Methods for Flexural Properties of Unreinforced and Reinforced Plastics and Electrical Insulating Materials; ASTM D790-17; ASTM International: West Conshohocken, PA, USA, 2017.

9. Nobile, M.R.; Acierno, D.; Incarnato, L.; Nicolais, L. The rheological behavior of a polyetherimide and of its blends with a thermotropic copolyester. J. Rheol. 1990, 34, 1181-1197. [CrossRef]

10. Ramiro, J.; Eguiazabal, J.I.; Nazabal, J. Synergistic mechanical behaviour and improved processability of poly(ether imide) by blending with poly(trimethylene terephthalate). Polym. Adv. Technol. 2003, 14, 129-136. [CrossRef]

11. Lin, B.; Sundararaj, U. Visualization of Poly (ether imide) and Polycarbonate Blending in an Internal Mixer. J. Appl. Polym. Sci. 2004, 92, 1165-1175. [CrossRef] 
12. Chun, Y.S.; Lee, H.S.; Kim, W.N.; Oh, T.S. Thermal properties and morphology of blends of poly(ether imide) and polycarbonate. Polym. Eng. Sci. 1996, 36, 2694-2702. [CrossRef]

13. Ramiro, J.; Eguiazabal, J.I.; Nazábal, J. Compatibilized Poly(ether imide)/Amorphous Polyamide Blends by Means of Poly(ethylene terephthalate) Addition. Polym. Eng. Sci. 2006, 46, 1292-1298. [CrossRef]

14. Cicala, G.; Latteri, A.; Del Curto, B.; Lo Russo, A.; Recca, G.; Farè, S. Engineering thermoplastics for additive manufacturing: A critical perspective with experimental evidence to support functional applications. J. Appl. Biomater. Funct. Mater. 2017, 15, 10-18. [CrossRef]

15. Ramiro, J.; Eguiazabal, J.I.; Nazabal, J. Phase structure and mechanical properties of blends of poly(ether imide) and bisphenol A polycarbonate. Polym. J. 2004, 36, 705-715. [CrossRef]

16. 3dxtech. Available online: https://www.3dxtech.com/ultem-9085-3d-printing-filament (accessed on 10 Febraury 2018).

(C) 2018 by the authors. Licensee MDPI, Basel, Switzerland. This article is an open access article distributed under the terms and conditions of the Creative Commons Attribution (CC BY) license (http:// creativecommons.org/licenses/by/4.0/). 